\title{
Lactobacilli sp. mixture alleviates LPS-induced inflammation in Caco-2 intestinal cell line
}

\author{
Gina Cecilia Pistol ${ }^{* 1}$ and Ionelia Taranu ${ }^{1}$ \\ *Corresponding author: gina.pistol@ibna.ro
}

${ }^{1}$ Laboratory of Animal Biology, National Institute of Research and Development for Biology and Animal Nutrition, INCDBNA-IBNA Balotesti, Romania

\section{ABSTRACT}

In intestinal inflammation disorders (inflammatory bowel diseases, IBD), the strategies of chronic inflammation management are oriented to the alternative therapies. There were demonstrated the beneficial effects of probiotics as modulators of intestinal inflammation. The present study aimed to investigate the effects of a probiotic Lactobacilli mixture on proinflammatory cytokines and in-depth MAPK signalling pathway in an in vitro model of intestinal inflammation. Intestinal Caco-2 cells were stimulated with bacterial lipopolysaccharide (LPS) for 4 hours; cells were cultured in presence of Lactobacilli sp. ( $L b$ ) mixture (Lb rhamnosus, Lb. paracasei and $L b$. acidophilus, $1 \times 10^{8} \mathrm{CFU}$ each $L b$ ) for additional 24 hours. Genomic and proteomic analyses were performed to evaluate 22 inflammatory-related genes and proteins (cytokines and their receptors) and p38/JNK/ERK MAP kinases. The Lactobacilli mixture inhibited the pro-inflammatory cytokines expression in LPS-treated Caco- 2 cells, the most affected cytokines being $T N F-\alpha$ and $I L-12 \quad p 70$ and up-regulated the anti-inflammatory cytokines $I L-4$ and $I L-10$ genes and proteins when compared to LPS-stimulated. A percent of $66 \%$ of genes and $60 \%$ of MAPKs proteins were down-regulated by $L b$ mixture, under the level of LPS-treated cells. Our data suggest that Lactobacilli mixture might inhibit pro-inflammatory cytokines via p38/JNK/ERK MAPKs signalling pathways in LPS-stimulated Caco-2 cells.

Keywords: Caco-2 cells; intestinal inflammation; probiotics; signalling pathways.

\section{INTRODUCTION}

The inflammatory bowel diseases (IBD) are immune-mediated diseases, characterized by acute or chronic intestinal inflammation, with pathogenic features and in-depth mechanisms still incomplete studied (Mowat et al, 2011). The therapeutic agents used for IBD therapy/treatment were 
associated with a number of potential risks (including infection, local reactions, malignancy (McLean and Cross, 2014) and with loss of response over time (Ben-Horin and Chowers, 2011). In the last decades, an increased attention was oriented to the alternative therapies, used to control symptoms and to manage chronic inflammation in IBD (Cheifetz et al, 2017). These alternatives are derived from a number of natural sources, including prebiotics, dietary supplements and probiotics (Hilsden et al, 2011).

Probiotics are defined, according to the World Health Organization, as "microorganisms that, when administered in adequate amounts confer a health benefit on the host" (FAO/WHO, 2006). The beneficial effects of probiotics, including Lactobacilli and Bifidobacteria sp., are exerted through different mechanisms such as antimicrobial peptide production, immune modulation, improvement of intestinal epithelial barrier integrity and function, leading to the potential attenuation of intestinal inflammation and to the reinforcement of epithelium functionality and structure (La Fata et al, 2018). They can also regulate both innate and adaptative immune response, stimulating anti-inflammatory reactions and modulating the microbiota dynamics and composition (La Fata et al, 2018). The potential use of probiotics in prevention or treatment of colitis was intense studied, in both cellular and animal models and also in IBD patients (Dieleman et al, 2003; Owczarek et al, 2016). In vitro studies demonstrated that Lactobacillus rhamnosus GG strain could prevent the cytokine-induced apoptosis in mouse or human colon cells (Yan and Polk, 2002). The same authors demonstrated that the Lb rhamnosus effects were modulated via anti-apoptotic Akt/PKB and p38 MAPK signaling pathways. Due to the strain-dependent effects of probiotics, in the last years studies were focused on the investigation of probiotic mixtures in intestinal inflammation (Bonavina et al, 2019; Liu et al, 2019). Our previous studies demonstrated the increased potential of probiotic Lactobacilli sp. mixtures in reducing intestinal inflammation, arguing that these mixtures could be more effective than a single strain (Pistol et al, 2019; Pistol et al, 2020; Taranu et al, 2018).

The positive effect of probiotic mixtures in IBD patients was already demonstrated, a high-concentration probiotic mixture of Lactobacilli, Bifidobacterium and Streptococcus strains, named VSL\#3, being used as commercial therapeutic option for IBD (Chapman et al, 2006 Liu et al, 2019). Although is encouraged the use of probiotics in the cure or prevention of intestinal inflammation, the main challenge in probiotic research is to decipher the in-depth mechanism of action and mediators of probiotic actions (Yan and Polk, 2020). Taking into account these data, the present study aimed to investigate the effects of a probiotic Lactobacilli mixture on pro-inflammatory cytokines and in-depth MAPK signaling pathway in an in vitro model of intestinal inflammation. We evaluated the effect of the mixture on Caco-2 cell monolayer exposed to LPS, and genomic and proteomic 
analyses were performed to evaluate the effects of Lactobacilli mixture on 22 inflammatory-related genes and proteins (cytokines and their receptors) and on p38/JNK/ERK members of MAPK signaling pathway.

\section{MATERIALS AND METHODS}

\section{Cell culture and treatments:}

The Lactobacillus (Lb) rhamnosus (ID IBNA02), Lb. paracasei (ID 13239) and $L b$. acidophilus (ID 11692) strains were cultured in MRS (DeManRogosa and Sharpe) broth (Sigma, Germany) at $37^{\circ} \mathrm{C}$ for $16 \mathrm{~h}$. The cultured Lb were diluted 1:10 in fresh MRS medium and were cultured for supplementary 4 hours, to achieve the logarithmic growing phase. The bacterial cell counts were estimated by the measurement of optical density at $600 \mathrm{~nm}$. The $\mathrm{Lb}$ cells were collected by centrifugation (10 minutes, $\left.4000 \mathrm{rpm}, 4^{\circ} \mathrm{C}\right)$ and resuspended in Minimum Essential Medium (MEM) culture medium, without antibiotic. The resulted Lb mixture had a concentration of $3 \times 108 \mathrm{CFU} / \mathrm{mL}$ total $\mathrm{Lb}(1 \mathrm{x} 108 \mathrm{CFU} / \mathrm{mL}$ of each strain).

Confluent Caco-2 human intestinal cells were cultured and maintained in MEM supplemented with 10\% Foetal Bovine Serum, 1\% antibiotic and 1\% LGlutamine at $37 \mathrm{C}$ in a 5\% CO2 humidified atmosphere. For the induction of inflammation, the cells were stimulated with $5 \mu \mathrm{g} / \mathrm{ml}$ of LPS, for 4 hours. Afterwards, the cells were incubated in the presence of Lb mixture (3x108CFU/mL total Lb) for $24 \mathrm{~h}$. All the experiments were performed in triplicates. At the end of the experiment, the cells were collected, washed with sterile PBS, lysed and used for array analyses. The collected supernatants were stored at $-80 \mathrm{C}$ until the protein array analyses.

\section{Quantitative PCR ( $($ PCR) array analysis}

Total RNA (tRNA) isolation: The cultured Caco-2 cells were homogenized in RTL buffer (QIAGEN GmbH, Germany) with an Ultra-Turrax homogenizer (IKA®- Werke GmbH \& KG, Germany) and tRNA was extracted using Qiagen RNeasy mini kit (QIAGEN GmbH, Germany), according to the manufacturer`s instructions. After extraction, the quantity and integrity of the extracted tRNAs were measured on a Nanodrop ND- 1000 spectrophotometer (Thermo Fischer Scientific, USA) and verified by agarose gel electrophoresis.

Complementary DNA ( $C D N A$ ) synthesis: The total RNA isolated from each sample was treated with a ribonuclease inhibitor (RNasin ${ }^{\circledR}$ Plus RNase Inhibitor Promega Corp., USA). The M-MLV Reverse Transcriptase kit (Life Technologies, USA) was used for cDNA synthesis, according to the manufacturer's protocol. The quality and purity of obtained cDNA was evaluated using GeneQuerry ${ }^{\mathrm{TM}}$ Human cDNA Evaluation Kit (ScienCell, USA), according to manufacturer`s protocol. 
qPCR: The customised qPCR array plates (GeneQuerry ${ }^{\mathrm{TM}}$ qPCR Array, ScienCell, USA) were used to evaluated the expressions of genes coding for 28 inflammatory $(I F N-\gamma, I L-1 \alpha, I L-1 \beta, I L-2, I L-3, I L-4, I L-6, I L-7, I L-8, I L-10, I L-$ 11, IL-12 p40, IL-12 p70, IL-13, IL-15, IL-16, IL-17, TNF- $\alpha, T N F-\beta, I L-6 s R, s$ TNF $R I, s$ TNF RII) and signalling markers (MAPKs: $p 38 \alpha, p 38 \delta$ MAPKs, JNK1, JNK2, $E R K 1$ and ERK2). The qPCR reaction set-up was already described in Pistol et al, 2019. A panel of five reference genes ( $\beta$-actin, GAPDH, LHDA, NONO, PPIH) was used, and for data normalisation were selected two reference genes (GAPDH and NONO), based on the NormFinder software selection. The ddCt method (Livak and Schmittgen, 2001) was used for the data analysis. The results were expressed as fold change (Fc) of treatments groups compared to un-stimulated control cells.

\section{Protein array analysis}

Two protein array analyses for detection of both inflammatory and signalling proteins were used. The levels of inflammatory markers in cell culture supernatants were measured using Inflammation Human Membrane Antibody Array (Abcam, UK), according to manufacturer`s recommendation. The markers used in this array were the corresponding proteins encoded by the genes enlisted in qPCR array analysis description. The phosphorylation level of signalling proteins (p38/JNK/ERK MAPKs, MKK, MSK and RSK kinases) was detected by using human Phospho-MAPK Array kit (R\&D Systems, USA), according to the manufacturer's protocol. The protein expressions were detected by chemiluminescence method and were developed using MicroChemi Imager (DNR Bio-Imaging Systems LTD, Israel). The obtained results were normalised to a positive control signal intensity and the relative expression of treatments groups were compared to the Control cells.

\section{Statistical analysis}

The results are expressed as mean \pm standard error of the mean (SEM). One way ANOVA analysis was used to test the differences among groups (StatView software 6.0, SAS Institute, USA) and $\mathrm{p}<0.05$ was considered statistically significant.

\section{RESULTS}

1. The effects of Lb mixture on the genes coding for inflammatory markers in LPS-treated Caco-2 cells

Cytokines are key mediators with important role in the initiation and perpetuation of inflammatory reaction (Friedrich et al, 2019). We used a previously established in vitro model of intestinal inflammation (LPS-treated Caco-2 cells) (Pistol et al, 2019) to assess the effect of Lb mixture on the 
cytokine levels. The expression of 22 inflammatory-related genes (cytokines and their receptors) was assessed by qPCR array analysis. As shown in Table 1, LPS treatment leaded to up-regulation of all pro-inflammatory cytokine genes $(17 / 17)$ when compared to untreated cells (Table 1$)$, the most affected genes being $I L-2(+20 \mathrm{Fc}$ increase, $p=0.015$ vs Control), $I L-12 p 40(+16 \mathrm{Fc}$ increase, $p=0.025 v s$ Control) and $I F N-\gamma(+15 \mathrm{Fc}$ increase, $p=0.002$ vs Control). Also, LPS treatment reduced the $I L-10$ anti-inflammatory gene expression ($50 \%$ reduction, $p=0.019$ vs Control, Table 1 ). The treatment with $L b$ mixture induced also an overexpression of all analysed genes coding for inflammatory cytokines (Table 1 ). In this case, the most affected mRNAs were $I L-1 \beta(+28 \%$ increase, $p=0.009$ vs Control), $I L-2(+17 \%$ increase, $\mathrm{p}=$ 0.050 vs Control) and $I F N-\gamma(+16 \%$ increase, $p=0.018$ vs Control) (Table 1). The addition of $L b$ mixture to LPS-treated Caco- 2 cells induced a decrease of mRNA levels of $23 \%(4 / 17)$ when compared to LPS-stimulated cells. Of these, TNF- $\alpha$ and $I L-12 p 70$ gene expressions were the most affected by Lb mixture (TNF- $\alpha$ : $-33 \%, p=0.008$ vs LPS group; $I L-12 p 70:-16 \%, p=0.037$ vs LPS group, Table 1).

When the genes coding for receptors for soluble form of cytokines were analysed, the results showed an up-regulation of all these genes $(3 / 3)$ induced by LPS treatment (Table 1). $L b$ mixture added to the untreated cells induced an overexpression of all receptor for cytokines mRNAs when compared to Control cells and also of $66.7 \%$ (2/3 genes, sTNFRI and sTNFRII) reported to LPS-treated cells. (Table 1).

2. The effects of Lb mixture on the inflammatory protein expressions in LPS-treated Caco-2 cells

Next, we analysed by protein array technique the protein expressions of the selected inflammatory markers. The results obtained, presented in Table 2, were similar with those described for gene expressions. Briefly, LPS treatment induced a strong inflammatory response in Caco-2 cells, by the increase of all analysed pro-inflammatory cytokines expression $(17 / 17$, Table 2) and by decreasing the anti-inflammatory IL-4 and IL-10 protein level in Caco-2 cells supernatant (Table 2). Lb mixture added to untreated cells induced an up-regulation of $64.7 \%$ (11/17) of proinflammatory cytokines compared to Control cells (Table 2). The treatment of LPSstimulated Caco- 2 cells with $L b$ mixture leaded to the downregulation of $29 \%(5 / 17)$ of pro-inflammatory cytokines protein expression and to the upregulation of IL-4 and IL-10 anti-inflammatory proteins when compared to LPS group (Table 2). The treatment of untreated Caco-2 cells with $L b$ mixture alone leaded to an increase of IL-6sR protein over the Control level $(+41 \%$ increase, $p=0.004$ vs Control, Table 2); the addition of $L b$ mixture to LPS- 
stimulated cells cannot reduce the increasement of genes coding for cytokines receptors below the level of LPS group (Table 2).

3. The effects of Lb mixture on the genes coding for signalling markers in LPS-treated Caco-2 cells

As important in-depth mediators of inflammation, the MAPK members (p38, JNK and ERK kinases) controls the expression of major cytokines and chemokines (Coskun et al, 2011). The effects of cellular treatments on the genes coding for $p 38 / J N K / E R K M A P K s$ were evaluated by qPCR analysis. Our results presented in Figure 1 showed that LPS challenge induced an upregulation of all analysed $p 38 / J N K / E R K M A P K$ genes. Of these, the most overexpressed genes were ERK2 (3.75-fold increase, $p=0.006$ vs Control cells, Figure 1) and $p 38 \delta$ (3.51-fold increase, $p=0.048$ vs Control, Figure 1). The addition of $L b$ mixture to untreated cells induced an up-regulation of 4 2/6 MAPK genes, ( $p 38 \alpha$ : 2-fold increase, $p=0.008 v s$ Control; ERK2: 3-fold increase, $p=0.050$ vs Control, Figure 1). In LPS-stimulated Caco-2 cells, the treatment with $L b$ mixture reduced the JNK/ERK mRNAs to the Control level (Figure 1).

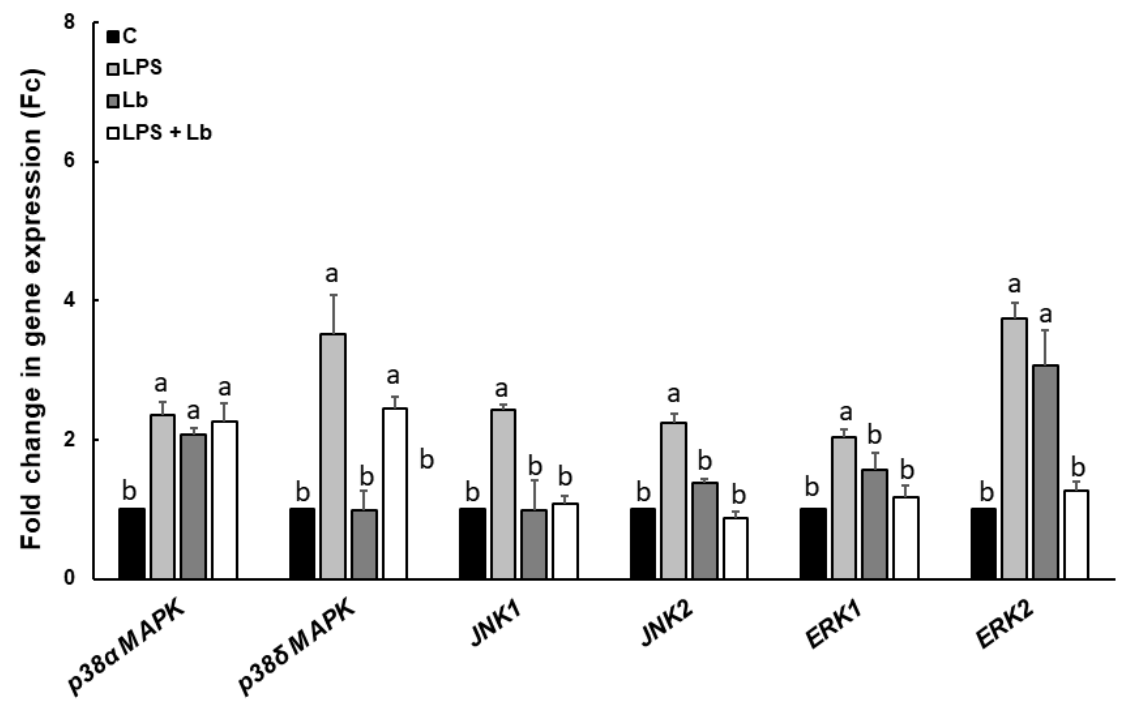

Figure 1. The effects of Lactobacillus mixture on p38/JNK/ERK MAPK genes in LPStreated Caco-2 cells. After treatment with LPS $5 \mu \mathrm{g} / \mathrm{ml}$, for 4 hours, Caco-2 cells were cultured in presence of Lactobacillus $(\mathrm{Lb})$ mixture $\left(3 \times 10^{8} \mathrm{CFU} / \mathrm{mL}\right.$ total Lb) for 24 hours. The bars represent average \pm SEM for three independent experiments. $\mathrm{a}, \mathrm{b}=$ Mean values within $\mathrm{a}$ column with unlike superscript letters were significantly different $(\mathrm{p}<0.05)$. 
Table 1. The effect of $L b$ mixture on cytokine gene expression in LPS-treated Caco-2 cells

\begin{tabular}{|c|c|c|c|c|c|c|c|c|c|c|}
\hline \multirow{3}{*}{$\begin{array}{l}\text { Functional } \\
\text { classification }\end{array}$} & \multirow{3}{*}{ Gene Name } & \multicolumn{8}{|c|}{ Experimental group* } & \\
\hline & & \multirow{2}{*}{$\begin{array}{c}\text { C } \\
\text { Fc (average } \\
\pm \text { SEM) }\end{array}$} & \multicolumn{2}{|c|}{ LPS } & \multicolumn{3}{|c|}{$L b$} & \multicolumn{3}{|c|}{$L P S+L b$} \\
\hline & & & $\begin{array}{c}\text { Fc (average } \\
\pm \text { SEM) }\end{array}$ & $\begin{array}{l}\text { regulation } \\
\text { vs Control } \\
\end{array}$ & $\begin{array}{c}\text { Fc (average } \\
\pm \text { SEM) }\end{array}$ & $\begin{array}{l}\text { regulation } \\
\text { vs Control }\end{array}$ & $\begin{array}{l}\text { regulation } \\
\text { vs LPS }\end{array}$ & $\begin{array}{c}\text { Fc (average } \\
\pm \text { SEM) } \\
\end{array}$ & $\begin{array}{l}\text { regulation } \\
\text { vs Control } \\
\end{array}$ & $\begin{array}{c}\text { regulation } \\
\text { vs LPS }\end{array}$ \\
\hline \multirow{19}{*}{ cytokines } & $I F N-\gamma$ & $1.0 \pm 0.0^{c}$ & $15.1 \pm 0.5^{b}$ & up & $16.6 \pm 2.1^{b}$ & up & - & $23.7 \pm 1.1^{\mathrm{a}}$ & up & up \\
\hline & $I L-1 \alpha$ & $1.0 \pm 0.0^{\mathrm{c}}$ & $8.8 \pm 0.9 \mathrm{a}$ & up & $2.5 \pm 0.3^{b}$ & up & down & $6.6 \pm 0.7 \mathrm{a}$ & up & down \\
\hline & $I L-1 \beta$ & $1.0 \pm 0.0 \mathrm{~d}$ & $12.6 \pm 4.3^{c}$ & up & $28.8 \pm 2.2^{b}$ & up & up & $48.8 \pm 7.9$ a & up & up \\
\hline & $I L-2$ & $1.0 \pm 0.0^{\mathrm{a}}$ & $20.8 \pm 2.4^{b}$ & up & $17.1 \pm 4.0^{b}$ & up & - & $47.3 \pm 1.4^{\mathrm{a}}$ & up & up \\
\hline & $I L-3$ & $1.0 \pm 0.0^{c}$ & $4.4 \pm 1.7 \mathrm{~b}$ & up & $14.0 \pm 2.9 \mathrm{a}$ & up & up & $12.6 \pm 0.4^{\mathrm{a}}$ & up & up \\
\hline & $I L-4$ & $1.0 \pm 0.0^{c}$ & $1.1 \pm 0.02^{c}$ & - & $4.3 \pm 0.7^{b}$ & up & up & $10.3 \pm 1.7$ a & up & up \\
\hline & $I L-6$ & $1.0 \pm 0.0^{c}$ & $7.9 \pm 1.5^{b}$ & up & $5.5 \pm 0.9^{b}$ & up & down & $17.7 \pm 1.2^{\mathrm{a}}$ & up & up \\
\hline & $I L-7$ & $1.0 \pm 0.0 \mathrm{~b}$ & $6.9 \pm 1.1^{\mathrm{a}}$ & up & $5.3 \pm 0.6^{\mathrm{a}}$ & up & - & $5.4 \pm 0.7 \mathrm{a}$ & up & - \\
\hline & $I L-8$ & $1.0 \pm 0.0^{c}$ & $14.2 \pm 1.7^{\mathrm{a}}$ & up & $2.4 \pm 0.1^{b}$ & up & down & $17.2 \pm 2.8^{a}$ & up & - \\
\hline & $I L-10$ & $1.0 \pm 0.0^{\mathrm{a}}$ & $0.5 \pm 0.2^{b}$ & down & $1.2 \pm 0.2^{\mathrm{a}}$ & - & up & $1.2 \pm 0.4^{\mathrm{a}}$ & - & up \\
\hline & $I L-11$ & $1.0 \pm 0.0^{b}$ & $5.8 \pm 0.5^{a}$ & up & $4.5 \pm 1.6^{\mathrm{a}}$ & up & - & $5.0 \pm 0.2^{a}$ & up & - \\
\hline & $I L-12 p 40$ & $1.0 \pm 0.0 \mathrm{~d}$ & $16.2 \pm 2.4^{b}$ & up & $5.2 \pm 0.5^{c}$ & up & down & $37.8 \pm 5.2^{a}$ & up & up \\
\hline & $I L-12$ p 70 & $1.0 \pm 0.0^{\mathrm{d}}$ & $13.6 \pm 1.4^{a}$ & up & $2.7 \pm 0.4^{c}$ & up & down & $11.4 \pm 0.4^{b}$ & up & down \\
\hline & $I L-13$ & $1.0 \pm 0.0 \mathrm{~d}$ & $11.6 \pm 1.7^{b}$ & up & $4.5 \pm 0.3^{c}$ & up & down & $25.0 \pm 2.4^{\mathrm{a}}$ & up & up \\
\hline & $I L-15$ & $1.0 \pm 0.0^{c}$ & $7.4 \pm 1.9^{a}$ & up & $5.2 \pm 0.4 \mathrm{~b}$ & up & down & $8.9 \pm 0.6^{a}$ & up & - \\
\hline & $I L-16$ & $1.0 \pm 0.0 \mathrm{~d}$ & $7.9 \pm 0.8^{b}$ & up & $5.4 \pm 1.5^{c}$ & up & down & $13.6 \pm 1.8^{a}$ & up & up \\
\hline & $I L-17$ & $1.0 \pm 0.0^{b}$ & $11.9 \pm 2.1^{\mathrm{a}}$ & up & $13.2 \pm 2.8^{a}$ & up & - & $13.7 \pm 2.4^{\mathrm{a}}$ & up & - \\
\hline & $T N F-\alpha$ & $1.0 \pm 0.0^{c}$ & $10.9 \pm 0.5^{\mathrm{a}}$ & up & $7.1 \pm 0.8^{b}$ & up & down & $7.3 \pm 0.6^{b}$ & up & down \\
\hline & $T N F-\beta$ & $1.0 \pm 0.0^{c}$ & $11.1 \pm 0.7 \mathrm{a}$ & up & $5.5 \pm 0.6^{b}$ & up & down & $9.6 \pm 1.2^{b}$ & up & down \\
\hline \multirow{3}{*}{$\begin{array}{l}\text { receptors for } \\
\text { soluble form of } \\
\text { cytokines }\end{array}$} & $I L-6 s R$ & $1.0 \pm 0.0^{c}$ & $4.7 \pm 1.2^{\mathrm{a}}$ & up & $2.2 \pm 0.4 \mathrm{~b}$ & up & down & $5.2 \pm 1.1^{\mathrm{a}}$ & up & - \\
\hline & $s$ TNF RI & $1.0 \pm 0.0^{c}$ & $2.5 \pm 0.4 \mathrm{~b}$ & up & $1.6 \pm 0.1 \mathrm{~b}$ & up & - & $4.9 \pm 1.2^{\mathrm{a}}$ & up & up \\
\hline & $s$ TNF RII & $1.0 \pm 0.0^{c}$ & $3.1 \pm 0.8^{b}$ & up & $0.9 \pm 0.3^{c}$ & - & down & $5.1 \pm 0.9$ a & up & up \\
\hline
\end{tabular}

${ }^{*} \mathrm{C}=$ untreated control cells; LPS = cells treated with ( $\left.5 \mu \mathrm{g} / \mathrm{ml} \mathrm{LPS}\right)$ for 4 hours; LPS $+L b$ mixture $=$ cells treated with $\mathrm{LPS}(5 \mu \mathrm{g} / \mathrm{ml})$ for $4 \mathrm{hours}$ and $L b \mathrm{mixture}\left(3 \mathrm{x} 10^{8}\right.$ total $L b$ ) for 24 hours; Fc= fold change in gene expression. Results are expressed as means \pm SEM of three independent experiments; the effect of the different treatments on cytokines gene expression was evaluated using ANOVA one-way test; a,b,c, $=$ Mean values within a row with unlike superscript letters were significantly different $(p<0.05)$. 
Table 2. The effect of $L b$ mixture on cytokine protein expression in LPS-treated Caco-2 cells

\begin{tabular}{|c|c|c|c|c|c|c|c|c|c|}
\hline \multirow{3}{*}{$\begin{array}{l}\text { Protein } \\
\text { Name }\end{array}$} & \multicolumn{8}{|c|}{ Experimental group* } & \\
\hline & \multirow{2}{*}{$\begin{array}{c}\text { C } \\
\text { MD (average } \pm \\
\text { SEM) }\end{array}$} & \multicolumn{2}{|c|}{ LPS } & \multicolumn{3}{|c|}{$L b$} & \multicolumn{3}{|c|}{ LPS $+L b$} \\
\hline & & $\begin{array}{c}\text { MD (average } \pm \\
\text { SEM)) }\end{array}$ & $\begin{array}{l}\text { regulation } \\
\text { vs Control }\end{array}$ & $\begin{array}{c}\text { MD (average } \pm \\
\text { SEM) }\end{array}$ & $\begin{array}{l}\text { regulation } \\
\text { vs Control }\end{array}$ & $\begin{array}{c}\text { regulation } \\
\text { vs LPS }\end{array}$ & $\begin{array}{c}\text { MD (average } \pm \\
\text { SEM)) }\end{array}$ & $\begin{array}{l}\text { regulation } \\
\text { vs Control }\end{array}$ & $\begin{array}{l}\text { regulation } \\
\text { vs LPS }\end{array}$ \\
\hline IFN- $\gamma$ & $156.2 \pm 14.4^{\mathrm{c}}$ & $1154.1 \pm 18.2^{\mathrm{a}}$ & up & $1016.5 \pm 16.4 \mathrm{~b}$ & up & down & $1011.6 \pm 40.1^{b}$ & up & down \\
\hline IL- $1 \alpha$ & $223.5 \pm 2.4^{c}$ & $1292.7 \pm 105.9$ a & up & $966.1 \pm 31.3^{b}$ & up & down & $941.0 \pm 53.9 \mathrm{~b}$ & up & down \\
\hline IL-1 $\beta$ & $2.0 \pm 0.1^{\mathrm{c}}$ & $1907.9 \pm 128.0^{a}$ & up & $332.2 \pm 67.6^{b}$ & up & down & $1957.9 \pm 80.8^{\mathrm{a}}$ & up & - \\
\hline IL-2 & $20.5 \pm 0.6^{d}$ & $578.9 \pm 38.0^{b}$ & up & $160.5 \pm 22.9^{c}$ & up & down & $1584.1 \pm 30.7$ a & up & up \\
\hline IL-3 & $186.4 \pm 22.8^{d}$ & $1543.1 \pm 102.2 \mathrm{~b}$ & up & $1007.7 \pm 59.8^{c}$ & up & down & $2375.2 \pm 99.7$ a & up & up \\
\hline IL-4 & $575.3 \pm 38.6^{b}$ & $168.2 \pm 34.7^{\mathrm{c}}$ & down & $509.8 \pm 65.2^{b}$ & - & up & $1563.2 \pm 314.2^{\mathrm{a}}$ & up & up \\
\hline IL-6 & $103.6 \pm 2.3^{c}$ & $4051.5 \pm 484.2 \mathrm{~b}$ & up & $108.6 \pm 6.2^{c}$ & - & down & $6067.7 \pm 223.8 \mathrm{a}$ & up & up \\
\hline IL-7 & $157.1 \pm 13.7 \mathrm{~b}$ & $587.2 \pm 41.7 \mathrm{a}$ & up & $186.7 \pm 30.8^{b}$ & - & down & $547.2 \pm 102.1^{\mathrm{a}}$ & up & - \\
\hline IL-8 & $826.6 \pm 116.8^{c}$ & $16564.2 \pm 353.4^{a}$ & up & $1080.0 \pm 298.5^{c}$ & - & down & $15003.3 \pm 280.0 \mathrm{~b}$ & up & down \\
\hline IL-10 & $1211.2 \pm 142.9^{a}$ & $609.1 \pm 46.0 \mathrm{~b}$ & down & $1192.4 \pm 88.6^{a}$ & - & up & $1213.7 \pm 142.6^{a}$ & - & up \\
\hline IL-11 & $31.7 \pm 9.1^{c}$ & $911.9 \pm 41.8^{a}$ & up & $452.9 \pm 46.1 \mathrm{~b}$ & up & down & $853.4 \pm 52.8^{a}$ & up & - \\
\hline IL-12 p40 & $510.3 \pm 8.7 \mathrm{~d}$ & $1111.7 \pm 40.5^{b}$ & up & $945.7 \pm 27.1^{c}$ & up & down & $1310.4 \pm 17.2^{\mathrm{a}}$ & up & up \\
\hline IL-12 p70 & $260.0 \pm 30.7 \mathrm{c}$ & $1270.5 \pm 63.9 \mathrm{a}$ & up & $792.5 \pm 80.9 \mathrm{~b}$ & up & down & $967.6 \pm 83.1 \mathrm{~b}$ & up & down \\
\hline IL-13 & $99.3 \pm 0.3^{c}$ & $538.1 \pm 54.9 \mathrm{~b}$ & up & $67.3 \pm 5.9^{d}$ & down & down & $938.5 \pm 97.8^{a}$ & up & up \\
\hline IL-15 & $7.3 \pm 0.3^{c}$ & $1158.4 \pm 95.7 \mathrm{~b}$ & up & $287.3 \pm 63.4^{c}$ & up & down & $1789.2 \pm 158.3^{a}$ & up & up \\
\hline IL-16 & $273.2 \pm 39.4 \mathrm{~b}$ & $1247.6 \pm 135.2^{a}$ & up & $186.4 \pm 9.1 \mathrm{~b}$ & - & down & $1539.8 \pm 308.5^{a}$ & up & - \\
\hline IL-17 & $75.6 \pm 14.9^{c}$ & $576.3 \pm 70.5 b$ & up & $20.3 \pm 6.7^{d}$ & down & down & $1176.0 \pm 90.3^{a}$ & up & up \\
\hline TNF- $\alpha$ & $49.4 \pm 5.0 \mathrm{~d}$ & $2323.5 \pm 141.8^{a}$ & up & $131.1 \pm 6.7^{c}$ & up & down & $1382.1 \pm 244.0^{b}$ & up & down \\
\hline TNF- $\beta$ & $268.6 \pm 2.0^{c}$ & $2257.8 \pm 301.1^{b}$ & up & $887.1 \pm 108.6^{\mathrm{c}}$ & up & down & $3060.4 \pm 580.3^{a}$ & up & up \\
\hline IL-6sR & $1571.8 \pm 257.1^{\mathrm{d}}$ & $1881.3 \pm 7.9^{c}$ & up & $2221.1 \pm 115.3^{b}$ & up & up & $3448.8 \pm 301.4^{\mathrm{a}}$ & up & up \\
\hline s TNF RI & $1231.8 \pm 95.7^{c}$ & $1566.4 \pm 233.5^{b}$ & up & $1403.1 \pm 92.9^{c}$ & - & - & $3579.4 \pm 387.6^{\mathrm{a}}$ & up & up \\
\hline s TNF RII & $956.3 \pm 29.7 \mathrm{c}$ & $2037.5 \pm 23.9 \mathrm{~b}$ & up & $1173.6 \pm 71.0^{c}$ & - & down & $3490.8 \pm 277.3^{a}$ & up & up \\
\hline
\end{tabular}

${ }^{*} \mathrm{C}=$ untreated control cells; LPS = cells treated with $(5 \mu \mathrm{g} / \mathrm{ml} \mathrm{LPS})$ for 4 hours; $\mathrm{LPS}+L b$ mixture $=$ cells treated with $\mathrm{LPS}(5 \mu \mathrm{g} / \mathrm{ml})$ for 4 hours and $L b$ mixture $\left(3 \times 10^{8}\right.$ total $\left.L b\right)$ for 24 hours; MD= Mean density of protein expression. Results are expressed as means \pm SEM of three independent experiments and the effect of the different treatments on cytokine protein expression was evaluated using ANOVA one-way test; a,b,c,d = Mean values within a row with unlike superscript letters were significantly different $(p<0.05)$. 
Table 3. The effect of $L b$ mixture on signalling protein expression in LPS-treated Caco- 2 cells

\begin{tabular}{|c|c|c|c|c|c|c|c|c|c|}
\hline \multirow{3}{*}{$\begin{array}{l}\text { Protein } \\
\text { Name }\end{array}$} & \multicolumn{8}{|c|}{ Experimental group* } & \multirow[b]{3}{*}{$\begin{array}{c}\text { regulation } \\
\text { vs LPS } \\
\end{array}$} \\
\hline & \multirow{2}{*}{$\begin{array}{c}\text { C } \\
\text { MD (average } \\
\pm \text { SEM) }\end{array}$} & \multicolumn{2}{|c|}{ LPS } & \multicolumn{3}{|c|}{$L b$} & \multicolumn{2}{|c|}{$L P S+L b$} & \\
\hline & & $\begin{array}{l}\text { MD (average } \\
\pm \text { SEM) }\end{array}$ & $\begin{array}{l}\text { regulation } \\
\text { vs Control } \\
\end{array}$ & $\begin{array}{l}\text { MD (average } \\
\pm \text { SEM) } \\
\end{array}$ & $\begin{array}{l}\text { regulation } \\
\text { vs Control } \\
\end{array}$ & $\begin{array}{c}\text { regulation } \\
\text { vs LPS }\end{array}$ & $\begin{array}{c}\text { MD (average } \pm \\
\text { SEM) }\end{array}$ & $\begin{array}{l}\text { regulation } \\
\text { vs Control }\end{array}$ & \\
\hline p38 $\alpha$ MAPK & $1.3 \pm 0.1 \mathrm{~b}$ & $3.9 \pm 0.3 \mathrm{a}$ & up & $4.7 \pm 0.3$ a & up & - & $4.1 \pm 0.6^{\mathrm{a}}$ & up & - \\
\hline p38ß МАРК & $15.5 \pm 0.7^{c}$ & $21.6 \pm 0.8^{b}$ & up & $19.0 \pm 0.9 \mathrm{~b}$ & up & - & $25.5 \pm 0.5^{a}$ & up & up \\
\hline p38ठ MAPK & $15.0 \pm 0.9 b$ & $25.3 \pm 0.7 \mathrm{a}$ & up & $16.0 \pm 0.2^{a}$ & - & down & $11.3 \pm 0.4^{c}$ & down & down \\
\hline 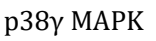 & $8.3 \pm 0.8^{c}$ & $17.8 \pm 0.6^{a}$ & up & $13.1 \pm 1.0 \mathrm{~b}$ & up & down & $6.5 \pm 0.6^{c}$ & - & down \\
\hline JNK1 & $1.9 \pm 0.3 \mathrm{~d}$ & $10.7 \pm 1.2^{\mathrm{a}}$ & up & $4.7 \pm 0.1^{c}$ & up & down & $7.4 \pm 0.8^{b}$ & up & down \\
\hline JNK2 & $16.0 \pm 0.7 \mathrm{~b}$ & $21.9 \pm 1.5^{\mathrm{a}}$ & up & $14.9 \pm 1.0^{b}$ & - & down & $20.6 \pm 0.8^{a}$ & up & - \\
\hline JNK3 & $8.0 \pm 1.7 \mathrm{~b}$ & $15.9 \pm 1.4^{\mathrm{a}}$ & up & $10.1 \pm 0.6^{b}$ & - & down & $9.7 \pm 1.1^{b}$ & up & down \\
\hline JNK pan & $16.9 \pm 0.5^{c}$ & $34.0 \pm 2.4^{\mathrm{a}}$ & up & $18.1 \pm 0.3^{c}$ & - & down & $21.9 \pm 1.1^{b}$ & up & down \\
\hline ERK1 & $4.2 \pm 0.6^{b}$ & $7.9 \pm 0.4^{\mathrm{a}}$ & up & $3.9 \pm 0.5^{b}$ & - & down & $2.9 \pm 0.4 \mathrm{~b}$ & - & down \\
\hline ERK2 & $5.1 \pm 0.3^{b}$ & $8.8 \pm 0.3$ a & up & $3.8 \pm 0.3^{c}$ & down & down & $7.7 \pm 0.7 \mathrm{a}$ & up & - \\
\hline MKK3 & $15.4 \pm 1.4^{\mathrm{c}}$ & $20.9 \pm 0.7$ & up & $15.4 \pm 1.4^{d}$ & down & down & $17.7 \pm 1.2^{b}$ & - & down \\
\hline MKK6 & $5.6 \pm 1.8^{b}$ & $11.6 \pm 0.6^{a}$ & up & $5.7 \pm 0.3^{b}$ & - & down & $6.9 \pm 1.2 b$ & - & down \\
\hline MSK2 & $15.7 \pm 1.8^{b}$ & $24.7 \pm 1.2^{\mathrm{a}}$ & up & $16.3 \pm 1.2^{b}$ & - & down & $14.5 \pm 2.0^{b}$ & - & down \\
\hline RSK1 & $9.2 \pm 0.8^{c}$ & $19.8 \pm 1.3^{a}$ & up & $12.6 \pm 0.7 \mathrm{~b}$ & up & down & $21.8 \pm 1.9^{a}$ & up & - \\
\hline RSK2 & $10.0 \pm 0.5^{b}$ & $15.6 \pm 0.7^{a}$ & up & $13.3 \pm 0.7^{\mathrm{a}}$ & up & - & $14.0 \pm 0.9^{a}$ & up & - \\
\hline
\end{tabular}

${ }^{*} \mathrm{C}=$ untreated control cells; LPS = cells treated with $(5 \mu \mathrm{g} / \mathrm{ml}$ LPS) for 4 hours; LPS $+L b$ mixture $=$ cells treated with LPS $(5 \mu \mathrm{g} / \mathrm{ml})$ for 4 hours and $L b$ mixture $(3 \times 10$ total $L b$ ) for 24 hours; MD= Mean density of protein expression. Results are expressed as means \pm SEM of three independent experiments; the effect of the different treatments on protein expression was evaluated using ANOVA one-way test; a,b,c,d = Mean values within a row with unlike superscript letters were significantly different 
4. The effects of Lb mixture on the signalling proteins in LPS-treated Caco2 cells

The results of signalling protein array analyses were similar with their corresponding genes results. LPS treatment increased the expressions of all analysed signalling proteins (15/15) compared to Control cells (Table 3). Compared to Control cells, $L b$ mixture alone up-regulated $40 \%(6 / 15)$ of the signalling proteins, especially $\mathrm{p} 38 \alpha / \beta / \gamma$ MAPKs (Table 3 ). When $L b$ mixture was added to the LPS-stimulated cells, a reduction of $60 \%$ of signalling proteins was accounted compared to LPS-stimulated cells (Table 3), while the p38 $\gamma$ MAPK, ERK1 MAPK, MKK3, MKK6 and MSK2 proteins were restored to the Control level (Table 3).

\section{DISCUSSIONS}

Probiotics have been proposed as a potential alternative for the prevention and/or treatment of the intestinal inflammatory diseases, and their effectiveness was demonstrated in both in vitro and in vivo models as well as in patients with IBD (Zaylaa et a, 2018). Probiotic supplements, particularly belonging to the Bifidobacterium and Lactobacillus genera, have been used in IBD patients (Saez-Lara et al, 2015), but their protective effects were strain-dependent. Also, several beneficial probiotic strains (Saccharomyces boulardii, Lactobacillus rhamnosus GG) have no effect in IBD, especially when applied in chronic patients (Lichtenstein et al, 2016). The current request is to understand the in-depth probiotic mechanisms in the intestinal cells, as well as to find the strains or mixtures of probiotics to be effective in counteract the intestinal inflammation.

In the present study, we investigated a mixture of 3 Lactobacillus strains using an in vitro model of intestinal inflammation, assessing both antiinflammatory capacities of this Lactobacillus mixture and the effects on the MAPKs signalling markers in LPS-treated Caco-2 cells.

We previously reported that the probiotic Lactobacillus sp. mixture could decrease the expressions of some inflammatory mediators (chemokines, growth factors and matrix metalloproteinases) in LPSstimulated cells, and these effects seemed to be regulated via PI3K/Akt, NF$k \mathrm{~B}$ and AhR signalling markers (Pistol et al, 2020). The effective mechanism of Lactobacilli mixture was demonstrated in the present study by inhibition of pro-inflammatory cytokines expression in LPS-treated Caco-2 cells, the most affected cytokines being TNF- $\alpha$ and $I L-12 p 70$, both at gene and protein level (Tables 1 and 2). Also, the anti-inflammatory cytokines $I L-4$ and $I L-10$ genes and proteins were up-regulated by $L b$ mixture addition, when compared to LPS-stimulated cells (Tables 1 and 2). Despite not all pro- 
inflammatory cytokines induced by LPS were down-regulated by Lactobacilli mixture treatment in our study, these results were in concordance with previous studies showing the differentially modulation of the proinflammatory cytokines by treatment with Lactobacillus strains in HT-29 and THP-1 cell lines (Duary et al, 2014; Aparna et al, 2013). Also, in a PBMCs/Caco-2 cells co-culture model stimulated with LPS (Haller et al, 2000 ), the effects of Lactobacillus strains were different and correlated with $L b$ strain used. Briefly, Lactobacillus sakei induced the $I L-1 \beta, I L-8$, and TNF- $\alpha$ proinflammatory cytokines expression, whereas Lactobacillus johnsonii increased the production of TGF- $\beta$ anti-inflammatory cytokine. These studies supported the hypothesis that the extent of anti-inflammatory effect of Lactobacillus was strain-dependent and moreover was affected by the time of the treatment.

In the pathogenesis of intestinal inflammation, activation of MAPKs and NF- $k$ B signalling pathways are key events, correlated, among others, with increased levels of pro-inflammatory cytokines (for example, TNF- $\alpha$ and IL6) (Atreya et al, 2008; Coskun et al, 2011). Previous studies reported also a down-regulation of MAPKs in cells treated with Lactobacilli sp. or with Lactobacilli components. In LPS-treated THP-1 cells, the addition of lipoteichoic acid from Lactobacillus plantarum was associated with a significant reduction of $\mathrm{p} 38, \mathrm{JNK} 1 / 2$ and ERK1/2 MAPK protein expressions (Kim et al, 2011). Also, in human colorectal cancer-derived HCT116 cell line, treatment with Lb. reuteri 6475 - derived histamine suppressed LPS-induced p38 MAPK activation (Shi et al, 2019). In a recent study, Qiao et al (2020) demonstrated the anti-inflammatory effects of Lactobacillus salivarius in ETEC K88-stimulated IPEC-J2 porcine cells, mediated via reduction of the p38MAPK and p65 NF- $k$ B phosphorylation (activation). Similarly, in our study a percent of $66 \%$ of genes coding for MAPKs and $60 \%$ of MAPKs proteins were down-regulated by the addition of $L b$ mixture, under the level from LPS-treated cells (Figure 1 and Table 3 ). In conclusion, our data suggest that Lactobacilli mixture consisted of Lb rhamnosus, $L b$. paracasei and $L b$. acidophilus might inhibit pro-inflammatory cytokines via the reduction of p38/JNK/ERK MAPKs expression in LPS-stimulated Caco-2 cells. Corroborated with our previous results reported in Pistol et al (2020), this reduction of the amplitude of inflammatory markers could be also regulated through inhibition of NF- $k$ B signalling.

\section{CONCLUSION}

Collectively, our results suggest that Lactobacilli mixture (Lb rhamnosus, $L b$. paracasei and $L b$. acidophilus) might reduce inflammation-related cytokines through inhibition of p38/JNK/ERK MAPK signaling pathway. This 
study may provide useful information for the use of Lactobacilli mixtures as potential strategies for the prevention/improvement of intestinal inflammation.

\section{ACKNOWLEDGEMENTS}

This research was supported by funds from the National Research Project PN-II-RU-TE-2014-4-1287 granted by the Romanian Ministry of Research and Innovation.

\section{REFERENCES}

Atreya, I., Atreya, R., Neurath, M.F., 2008. NF-kappaB in inflammatory bowel disease. J Intern Med. 263(6):591-6.

Ben-Horin, S., Chowers, Y., 2011. Review article: loss of response to anti-TNF treatments in Crohn's disease. Aliment Pharmacol Ther., 33(9): 98795.

Bonavina, L., Arini, A., Ficano, L., Iannuzziello, D., Pasquale, L., Aragona, S.E., Ciprandi, G., 2019. On Digestive Disorders ISG. Abincol® (Lactobacillus plantarum LP01, Lactobacillus lactis subspecies cremoris LLC02, Lactobacillus delbrueckii LDD01), an oral nutraceutical, pragmatic use in patients with chronic intestinal disorders. Acta Biomed. 90(7-S):8-12. doi: 10.23750/abm.v90i7S.8649.

Chapman, T.M., Plosker, G.L., Figgitt, D.P., 2006. VSL\#3 probiotic mixture: a review of its use in chronic inflammatory bowel diseases. Drugs, 66(10): 1371-87.

Cheifetz, A.S., Gianotti, R., Luber, R., Gibson, P.R., 2017. Complementary and alternative medicines used by patients with Inflammatory Bowel Diseases. Gastroenterology, 152(2): 415-429.

Coskun, M., Olsen, J., Seidelin, J.B., Nielsen, O.H., 2011. MAP kinases in inflammatory bowel disease. Clinica chimica acta; international journal of clinical chemistry, 412(7-8): 513-520.

Dieleman, L.A., Goerres, M. S., Arends, A., Sprengers, D., Torrice, C., Hoentjen, F., Grenther, W. B., Sartor, R. B., 2003. Lactobacillus GG prevents recurrence of colitis in HLA-B27 transgenic rats after antibiotic treatment. Gut, 52(3): 370-376.

Duary RK, Batish VK, Grover S., 2014. Immunomodulatory activity of two potential probiotic strains in LPS-stimulated HT-29 cells. Genes Nutr. 9(3):398. doi:10.1007/s12263-014-0398-2

FAO/WHO Organization., 2001. Probiotics in food: health and nutritional properties and guidelines for evaluation: Report of a Joint FAO/WHO Expert Consultation on Evaluation of Health and Nutritional Properties of Probiotics in Food including Powder Milk with Live 
Lactic Acid Bacteria, Cordoba, Argentina, 1-4 October 2001 [and] Report of a Joint FAO/WHO Working Group on Drafting Guidelines for the Evaluation of Probiotics in Food, London, Ontario, Canada, 30 April -1 May 2002. 2006.

Friedrich, M., Pohin, M., Powrie, F., 2019. Cytokine Networks in the Pathophysiology of Inflammatory Bowel Disease. Immunity, 50(4): 992-1006.

Haller, D., Bode, C., Hammes, W. P., Pfeifer, A. M. A., Schiffrin, E. J., and Blum, S., 2000. Non-pathogenic bacteria elicit a differential cytokine response by intestinal epithelial cell/leucocyte co-cultures, Gut, 47(1): 79-87

Hilsden, R.J., Verhoef, M.J., Rasmussen, H., Porcino, A., DeBruyn, J.C., 2011. Use of complementary and alternative medicine by patients with inflammatory bowel disease. Inflamm Bowel Dis, 17(2): 655-62.

Kim, H.G., Lee, S.Y., Kim, N.R., Lee, H.Y., Ko, M.Y., Jung, B.J., Kim, C.M., Lee, J.M., Park, J.H., Han, S.H., 2011. Lactobacillus plantarum lipoteichoic acid down-regulated Shigella flexneri peptidoglycan-induced inflammation Mol. Immunol., 48 (4), 382-391

La Fata, G., Weber, P., Mohajeri, M.H., 2018. Probiotics and the Gut Immune System: Indirect Regulation. Probiotics Antimicrob Proteins, 10(1): 11-21.

Lichtenstein, L., Avni-Biron, I., Ben-Bassat, O., 2016. Probiotics and prebiotics in Crohn's disease therapies. Best Practice \& Research. Clinical Gastroenterology, 30(1): 81-88. doi: 10.1016/j.bpg.2016.02.002

Liu, X.J., Yu, R., Zou, K.F., 2019. Probiotic Mixture VSL\#3 Alleviates Dextran Sulfate Sodium-induced Colitis in Mice by Downregulating $\mathrm{T}$ Follicular Helper Cells. Curr Med Sci.39(3):371-378. doi: 10.1007/s11596-019-2045-z.

Livak, K.J., Schmittgen, T.D., 2001. Analysis of relative gene expression data using real-time quantitative PCR and the 2(-Delta Delta $\mathrm{C}(\mathrm{T})$ ) Method. Methods, 25(4): 402-8.

Zaylaa, M., Al Kassaa, I., Alard, J., Peucelle, V., Boutillier, D., Desramaut, J., Dabboussi, F., Pot, B., Grangette, C., 2018. Probiotics in IBD: Combining in vitro and in vivo models for selecting strains with both anti-inflammatory potential as well as a capacity to restore the gut epithelial barrier, Journal of Functional Foods, 47, 304-315, https://doi.org/10.1016/j.jff.2018.05.029.

McLean, L.P., Cross, R.K., 2014. Adverse events in IBD: to stop or continue immune suppressant and biologic treatment. Expert Rev Gastroenterol Hepatol. 8(33): 223-40. doi: 10.1586/17474124.2014.881715.

Mowat, C., Windsor, A., Ahmad, T., Arnott, I., Driscoll, R., Mitton, S., Orchard, T., Rutter, M., Younge, L., Lees, C., Ho, G.T., Satsangi, J., Bloom, S., 2011. 
Guidelines for the management of inflammatory bowel disease in adults. Gut, 60(5): 571-607. doi: 10.1136/gut.2010.224154.

Owczarek, D., Rodacki, T., Domagała-Rodacka, R., Cibor, D., Mach, T., 2016. Diet and nutritional factors in inflammatory bowel diseases. World Journal of Gastroenterology, 22(3): 895-905. doi: 10.3748/wjg.v22.i3.895.

Pistol, G.C., Marin, D.E., Dragomir, C., Taranu, I., 2019. Synbiotic combination of prebiotic grape pomace extract and probiotic Lactobacillus sp. reduced important intestinal inflammatory markers and in-depth signalling mediators in lipopolysaccharide-treated Caco-2 cells. British Journal of Nutrition, 2019. 121 (3): 291-305.

Pistol, G.C., Marin, D.E., Taranu, I., 2020. A mixture of Lactobacillus sp. modulates the expression of inflammatory molecules, signalling kinases and nuclear receptors in LPS-treated Caco-2 cell culture model. Archiva Zootechnica, 23(1): 55-72.

Qiao, J., Sun., Z., Liang, D., Li, H., 2020. Lactobacillus salivarius alleviates inflammation via NF- $\kappa B$ signaling in ETEC K88-induced IPEC-J2 cells. J Anim Sci Biotechnol. 11:76. doi: 10.1186/s40104-020-00488-5.

Saez-Lara, M. J., Gomez-Llorente, C., Plaza-Diaz, J., Gil, A., 2015. The role of probiotic lactic acid bacteria and bifidobacteria in the prevention and treatment of inflammatory bowel disease and other related diseases: A systematic review of randomized human clinical trials. BioMed Research International, 2015: 505878. 10.1155/2015/505878.

Shi, Z., Fultz, R.S., Engevik, M.A., Gao, C., Hall, A., Major, A., Mori-Akiyama, Y., Versalovic, J., 2019. Distinct roles of histamine H1- and H2-receptor signaling pathways in inflammation-associated colonic tumorigenesis. Am J Physiol Gastrointest Liver Physiol. 316(1):G205G216. doi: 10.1152/ajpgi.00212.2018.

Taranu, I., Marin, D.E., Braicu, C., Pistol, G.C., Sorescu, I., Pruteanu, L.L., Berindan Neagoe, I., Vodnar, D.C., 2018. In Vitro transcriptome response to a mixture of Lactobacilli strains in intestinal porcine epithelial cell line. International Journal of Molecular Science, 19(7): 1923, doi: 10.3390/ijms19071923

Yan, F., Polk, D.B., 2002. Probiotic bacterium prevents cytokine-induced apoptosis in intestinal epithelial cells. J Biol Chem., 277(52):5095965. doi: 10.1074/jbc.M207050200.

Yan, F., Polk, D.B., 2020. Probiotics and probiotic-derived functional factors-mechanistic insights into applications for intestinal homeostasis. Front. Immunol, 11:1428. doi: 10.3389/fimmu.20 